\title{
Pressurized intraperitoneal aerosol chemotherapy with oxaliplatin (PIPAC-OX) in patients with colorectal peritoneal metastases-a systematic review
}

\author{
Robin J. Lurvink ${ }^{1 \#}$, Koen P. Rovers ${ }^{1 \#}$, Simon W. Nienhuijs ${ }^{1}$, Geert-Jan Creemers ${ }^{2}$, Jacobus W. A. Burger ${ }^{1}$, \\ Ignace H. J. de Hingh ${ }^{1,3}$ \\ ${ }^{1}$ Department of Surgery, ${ }^{2}$ Department of Medical Oncology, Catharina Hospital, Eindhoven, The Netherlands; ${ }^{3}$ GROW-School for Oncology and \\ Developmental Biology, Maastricht University, Maastricht, The Netherlands \\ Contributions: (I) Conception and design: All authors; (II) Administrative support: All authors; (III) Provision of study materials or patients: All \\ authors; (IV) Collection and assembly of data: All authors; (V) Data analysis and interpretation: All authors; (VI) Manuscript writing: All authors; (VII) \\ Final approval of manuscript: All authors. \\ "These authors contributed equally to this work and share first authorship. \\ Correspondence to: Prof. Ignace H. J. de Hingh, MD, PhD. Department of Surgery, Catharina Hospital, Eindhoven, The Netherlands. \\ Email: Ignace.d.hingh@catharinaziekenhuis.nl.
}

\begin{abstract}
Pressurized intraperitoneal aerosol chemotherapy with oxaliplatin (PIPAC-OX) is increasingly used as a palliative treatment option for patients with colorectal peritoneal metastases (CPM). The present study aimed to systematically review all clinical studies reporting safety and efficacy outcomes of PIPACOX in patients with CPM. PubMed, EMBASE, The Cochrane Library, and CINAHL were systematically searched to identify all clinical studies that included at least one patient with CPM treated with PIPACOX and reported one of the following outcomes: adverse events, tumor response, quality of life, secondary cytoreductive surgery, progression-free survival, overall survival, and environmental safety of PIPAC-OX. Results were narratively described. Of 28 included studies, only 14 non-comparative studies separately reported at least one outcome of PIPAC-OX for CPM, of which only two studies specifically focused on this group. These 14 studies reported adverse events (5 studies), tumor response (5 studies), secondary cytoreductive surgery (4 studies), progression-free survival (1 study), overall survival (5 studies), and environmental safety (2 studies). Except for 5 studies (describing 26 patients), none of the included studies stratified their results for PIPAC-OX monotherapy and PIPAC-OX with concomitant systemic therapy, and none of the studies reporting survival outcomes stratified results for line of palliative treatment, complicating interpretation. No PIPAC-OX related deaths were reported. No occupational platinum was detected during PIPAC-OX. The available evidence regarding PIPAC-OX for CPM is limited and difficult to interpret. Despite these limitations, PIPAC-OX appears safe in patients with CPM and safe for operating personnel. To increase insight in the role of PIPAC-OX in this setting, investigators of ongoing and future studies are encouraged to report separate outcomes of PIPAC-OX for CPM, to stratify their results for PIPAC-OX monotherapy and PIPAC-OX with concomitant systemic therapy, and to stratify survival results for line of palliative treatment.
\end{abstract}

Keywords: Pressurized intraperitoneal aerosol chemotherapy (PIPAC); colorectal cancer; peritoneal metastases; oxaliplatin

Submitted Jul 03, 2020. Accepted for publication Sep 02, 2020.

doi: 10.21037/jgo-20-257

View this article at: http://dx.doi.org/10.21037/jgo-20-257 


\section{Introduction}

Peritoneal metastases are common in colorectal cancer patients (1). Most patients are treated with palliative systemic therapy, since they do not qualify for curative intent treatment with cytoreductive surgery with or without hyperthermic intraperitoneal chemotherapy (HIPEC) (2). However, patients with colorectal peritoneal metastases (CPM) gain less survival-benefit from systemic therapy than patients with colorectal liver or lung metastases, possibly related to a phenomenon called the "peritoneumplasma barrier", which results in lower chemotherapeutic concentrations in peritoneal metastases (3-5).

Intraperitoneal therapies have been developed to overcome this phenomenon. Pressurized intraperitoneal aerosol chemotherapy (PIPAC) is one of those new therapies. PIPAC is a laparoscopic method for repetitive delivery of low-dose intraperitoneal chemotherapy as a pressurized aerosol, claiming enhanced tumor penetration, homogeneous intraperitoneal drug distribution, and limited local and systemic toxicity (6-9). Due to these promising initial results, PIPAC is currently increasingly implemented in multiple centers worldwide $(10,11)$. In these centers, patients with CPM are treated with PIPAC with oxaliplatin (PIPAC-OX) in an empirically chosen dosage of $92 \mathrm{mg} / \mathrm{m}^{2}$ every 6-8 weeks with or without concomitant palliative systemic therapy.

To evaluate the available evidence regarding PIPAC-OX in patients with CPM, this study aims to systematically review all clinical studies that reported adverse events, tumor response, quality of life, progression-free (PFS) and overall (OS) survival, secondary cytoreductive surgery, and occupational safety in this subgroup. We present the following article in accordance with the PRISMA checklist (available at http://dx.doi.org/10.21037/jgo-20-257).

\section{Methods}

This systematic review was performed according to the Preferred Reporting Items for Systematic Review and Meta-Analyses (PRISMA) guidelines (12). Two researchers (KPR and RJL) independently performed the literature search, study selection, data collection, and data synthesis. In case of disagreement, a third investigator (IHJH) made the final decision.

\section{Eligibility criteria}

Original research papers were eligible if they included at least one patient with non-appendiceal CPM treated with PIPAC-OX and investigated at least one of the following outcomes: adverse events, radiological response, histopathological response, cytological response, macroscopic response, biochemical response, quality of life, PFS, OS, possibility for secondary cytoreductive surgery, or environmental safety. Studies that performed in vitro or in animal research were not considered eligible. Studies were not excluded based on language or publication date or publication status.

\section{Search}

On 1 July 2020, PubMed, EMBASE, CINAHL, and the Cochrane Library were systematically searched with the following search ("PIPAC" or "Pressurized Intraperitoneal Aerosol Chemotherapy"). No additional terms were added to the search to increase its sensitivity. The references of all eligible manuscripts were searched for additional eligible studies.

\section{Data collection and synthesis}

A standardized form was used for data collection and contained the following items: publication year, enrolment years, study setting, the total number of patients, the total number of patients with CPM treated with PIPAC-OX, the number of patients with CPM treated with PIPAC-OX who received previous palliative therapy, the total number of PIPAC-OX in patients with CPM, the number of patients with CPM treated with PIPAC-OX with concomitant systemic therapy, reported outcomes, outcome assessment, and whether outcomes were separately reported for patients with CPM receiving PIPAC-OX.

Further data extraction and synthesis was performed for all studies that separately reported at least one outcome in $\geq 1$ patient with CPM treated with PIPAC-OX. The following items were extracted from these studies for each outcome: the total number of patients with CPM treated with PIPAC-OX, the total number of patients with CPM treated with PIPAC-OX that were evaluable for outcome assessment, and the total number of PIPAC-OX that these patients received. 
The results of included studies were narratively described and grouped according to reported outcomes. No metaanalyses were performed due to the high degree of clinical heterogeneity.

\section{Results}

The study flowchart is shown in Figure 1. This review included 28 studies [14 studies proceeded to data synthesis (13-26), 14 studies excluded from data synthesis (27-40)]. An overview of the study characteristics, the CPM population within each study, the reported outcomes, outcome assessment, and whether the reported outcomes were stratified for patients with CPM, is shown in Table 1.

All included studies were retrospective observational studies or prospective cohort studies and were all published between 2015 and 2020. All studies included patients with unresectable peritoneal metastases. Among the included studies, the degree of clinical heterogeneity was very high - most studies included patients with peritoneal metastases from any primary tumor who were treated with PIPAC with various drugs, either as monotherapy or with concomitant systemic therapy, and either as first line or as later line of palliative treatment.

Out of 28 included studies, only 14 studies separately reported at least one outcome for $\geq 1$ patient with CPM treated with PIPAC-OX. Further data extraction and synthesis was performed in these 14 studies. In these 14 studies, the number of patients with CPM treated with PIPAC-OX ranged from 1 to 66 patients (median 13).

\section{Adverse events}

Adverse events were reported in 24 original manuscripts (13-22,27,28,31-39). Only five studies provided separate results for patients with CPM treated with PIPAC-OX $(13-17)$, of which one study (17) did not provide the exact numbers of adverse events and was therefore not included in the data synthesis (Table 2).

Three manuscripts reported adverse events [Common Terminology Criteria for Adverse Events (CTCAE), v.4] from in total 21 patients with CPM who received 48 PIPAC-OX (14-16). Four patients experienced CTCAE grade 3 abdominal pain (19\%), and no CTCAE grade 4 or 5 events occurred in these three studies. The following CTCAE grade $1-2$ events were reported: pain $(n=7,33 \%)$, nausea/vomiting $(n=7,33 \%)$, infection $(n=1,5 \%)$, diarrhea $(\mathrm{n}=1,5 \%)$, fever $(\mathrm{n}=4,19 \%)$, liver/renal toxicity $(\mathrm{n}=6,29 \%)$.
However, adverse events were not separately reported for patients treated with PIPAC-OX monotherapy and patients treated with PIPAC-OX with concomitant systemic therapy.

Also, one report provided a sub-analysis of their cohort, focusing on the occurrence of severe hypersensitivity reactions after repeated PIPAC-OX (13). Two CTCAE grade 4 severe hypersensitivity reactions occurred in $2 / 71$ (3\%) PIPAC-OX, which occurred during the second and third procedure. Both patients had received an oxaliplatincontaining regimen as part of previous palliative systemic therapy. Also, both patients received PIPAC-OX with concomitant systemic therapy, although not an oxaliplatinbased regimen.

\section{Radiological response}

Two original manuscripts reported radiological response $(18,20)$. Only one provided separate results for 15 patients with CPM, who in total received 45 PIPAC-OX (Table 3). All but one patient received two or more procedures, and a response evaluation was performed in these 14 patients by computed tomography (CT), although the definitions for CT assessment (e.g., Response Evaluation Criteria in Solid Tumors) were not provided. The study reported 5 (36\%) patients with progressive disease, $5(36 \%)$ patients with stable disease, and four patients $(28 \%)$ showed regression of disease. Twelve out of 14 (86\%) patients received PIPAC-OX with concomitant systemic therapy. The two patients that received PIPAC-OX monotherapy both had progressive disease on CT.

\section{Histopathological response}

Although histopathological response was reported in twelve studies (15-21,27-31), only five provided separate results for patients with CPM treated with PIPAC-OX (Table 4).

Four studies used the peritoneal regression grading scale (PRGS) to evaluate subsequent biopsies in 33/45 patients with CPM (16-19). Regressive disease was found in 19 (58\%) patients, stable disease in $10(30 \%)$ patients and progressive disease in $4(12 \%)$ patients. In three studies, 4 of 18 patients received PIPAC-OX monotherapy. In these four patients, regressive, stable, and progressive disease was found in one, two, and one patient, respectively. In the fourth study, it was unclear whether these 15 patients received PIPAC-OX monotherapy or PIPAC-OX with concomitant systemic therapy (17).

Finally, one study used the tumor response grading 


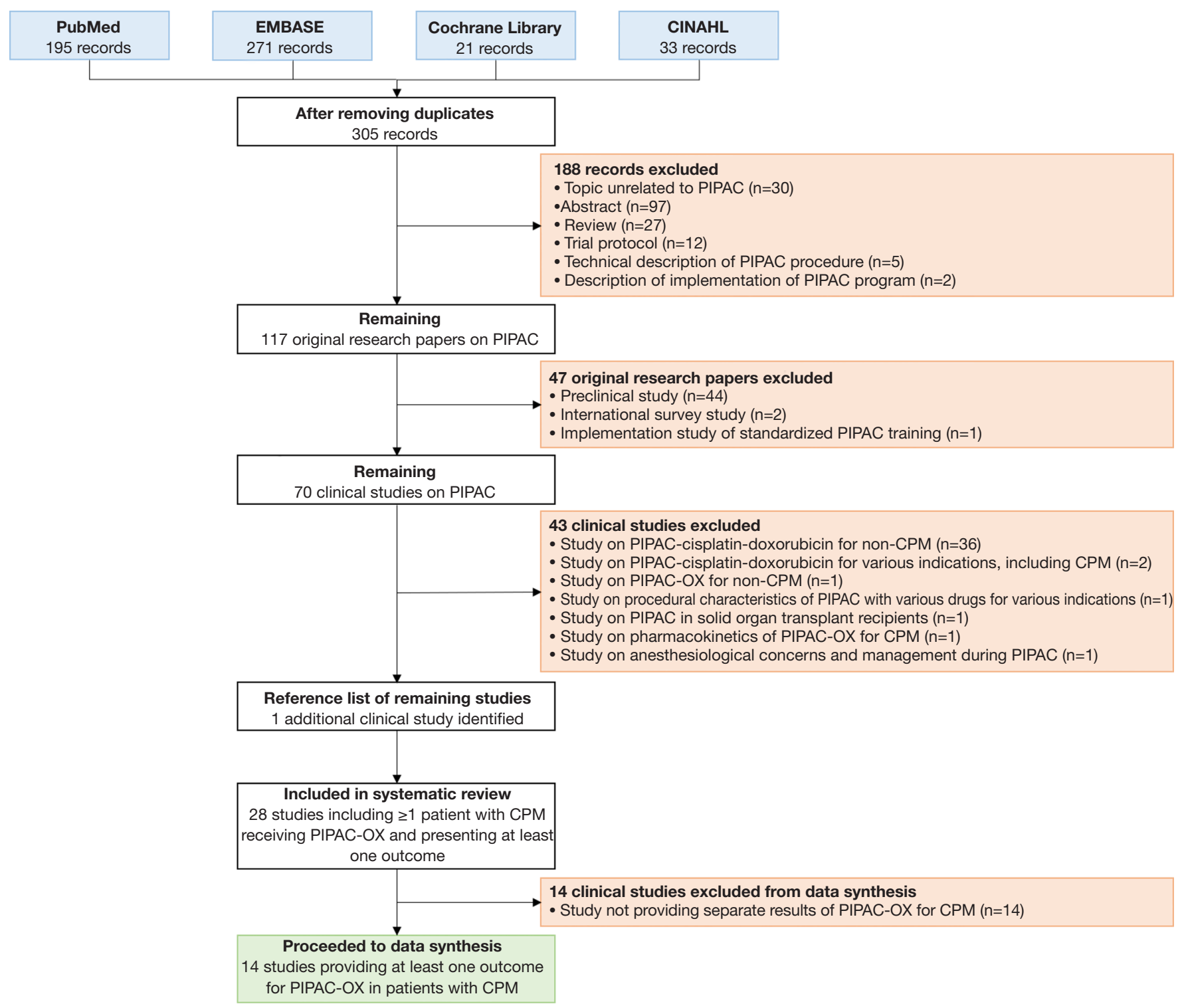

Figure 1 Literature search and study selection. CPM, Colorectal peritoneal metastases; PIPAC, pressurized intraperitoneal aerosol chemotherapy. Details of the literature search and study selection are presented in the Appendix.

system (15) (Table 5); 14 out of 17 patients with CPM were evaluable, and complete, major, partial, and no response was found in $7(50 \%), 4(29 \%), 1$ (7\%), and 2 (14\%) patients, respectively. However, results were not separately reported for patients receiving PIPAC-OX monotherapy and patients receiving PIPAC-OX with concomitant systemic therapy.

\section{Cytological response}

Five studies reported on cytological response in ascites or peritoneal lavage, of which three studies did not provide separate results for patients with CPM treated with PIPACOX (Table 6) $(17,19,28-30)$. The remaining two studies treated 27 patients with CPM with a total of 84 PIPAC-OX procedures $(17,19)$. Eighteen patients were evaluable for cytological response, which showed that $6(33 \%)$ patients converted from positive to negative cytology, cytology remained stable in $10(56 \%)$ patients, and $2(11 \%)$ patients converted from negative to positive cytology. However, one study did not separately report whether these patients were 







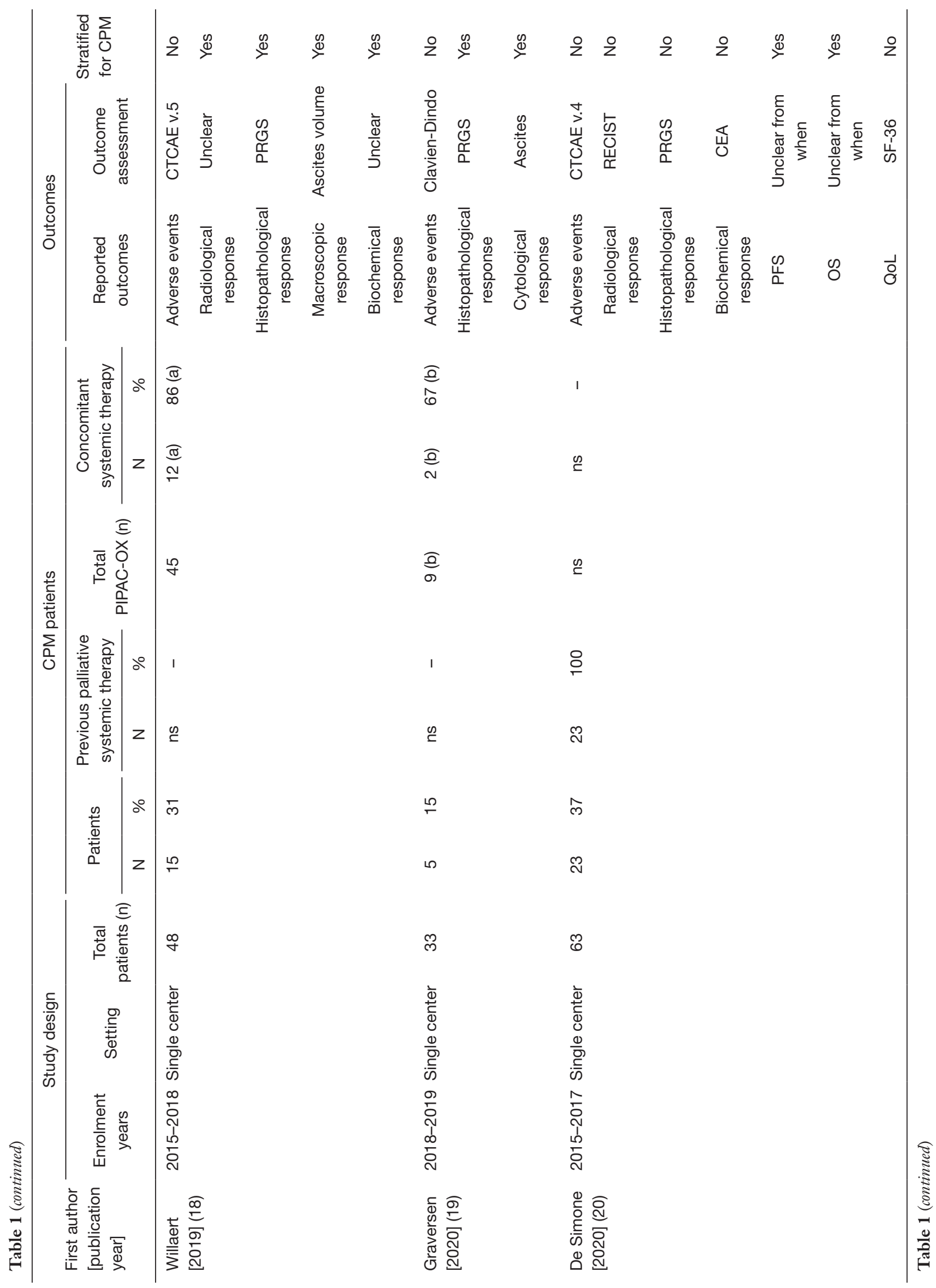

(C) Journal of Gastrointestinal Oncology. All rights reserved. $\quad$ J Gastrointest Oncol 2021;12(Suppl 1):S242-S258 I http://dx.doi.org/10.21037/jgo-20-257 


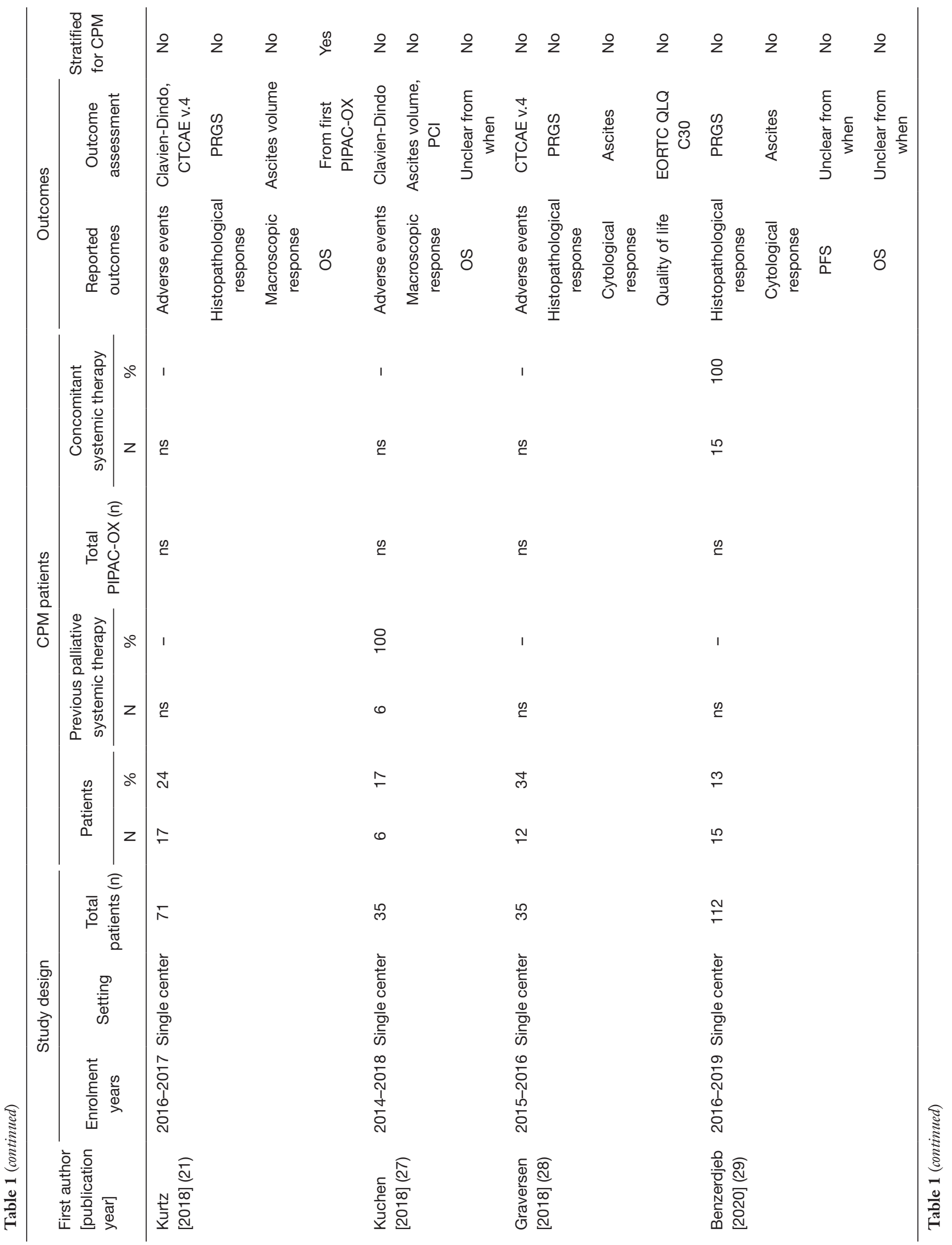




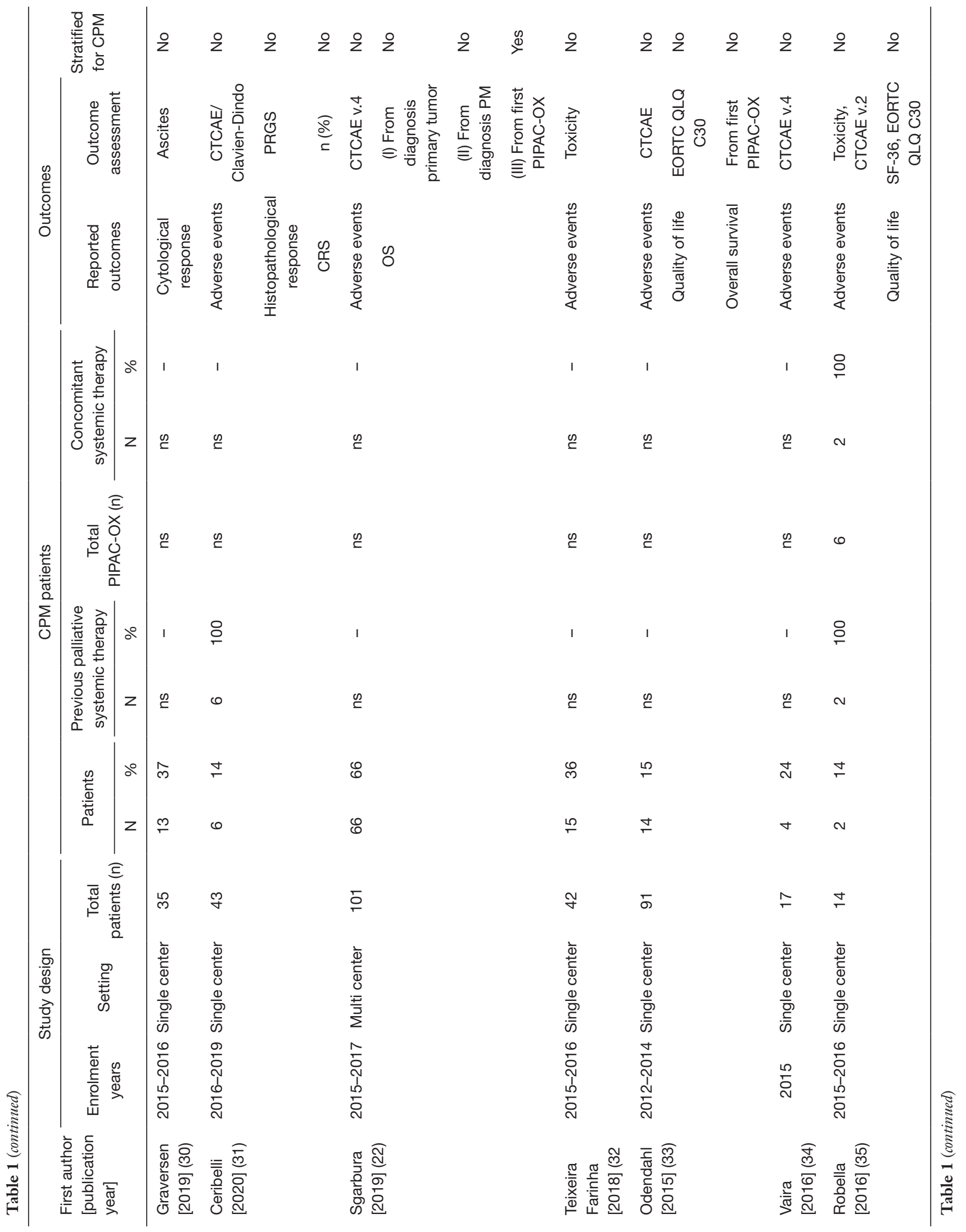




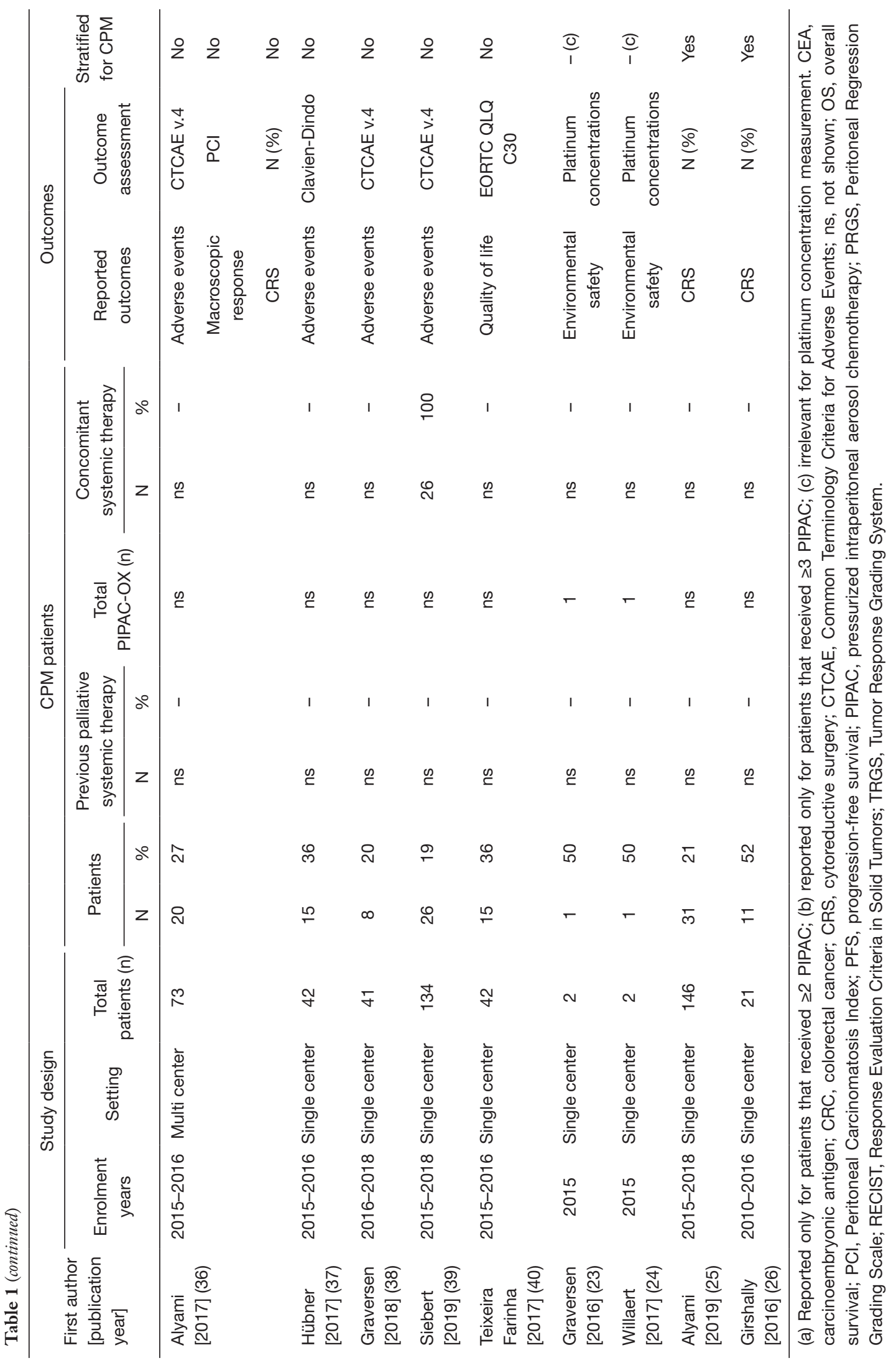


Table 2 Adverse events

\begin{tabular}{|c|c|c|c|c|c|c|c|}
\hline Studies & CRC patients & Evaluated patients & Total PIPAC-OX & \multicolumn{4}{|c|}{ CTCAE } \\
\hline Katdare [2019] (14) & 3 & 3 & 3 & 1 & 0 & 0 & 0 \\
\hline Demtröder [2016] (15) & 17 & 17 & 42 & 25 & 4 & 0 & 0 \\
\hline
\end{tabular}

CRC, colorectal cancer; PIPAC-OX, pressurized intraperitoneal aerosol chemotherapy with oxaliplatin; CTCAE, Common Terminology

Criteria for Adverse Events; ns, not shown.

Table 3 Radiologic response

\begin{tabular}{lccccc}
\hline \multirow{2}{*}{$\begin{array}{l}\text { Study } \\
\text { Willaert [2019] (18) }\end{array}$} & CRC patients & Evaluated patients & Total PIPAC-OX & \multicolumn{3}{c}{ Response assessment } \\
\cline { 5 - 7 } & 15 & 14 & Progressive & Stable & Regressive \\
\hline
\end{tabular}

$\mathrm{CRC}$, colorectal cancer; PIPAC-OX, pressurized intraperitoneal aerosol chemotherapy with oxaliplatin.

Table 4 Histopathological response-Peritoneal Regression Grading Score



(a) Reported for the 3 evaluated patients. CRC, colorectal cancer; PIPAC-OX, pressurized intraperitoneal aerosol chemotherapy with oxaliplatin.

Table 5 Histopathological response-Tumor Response Grading Scale

\begin{tabular}{lccccccc}
\hline \multirow{2}{*}{$\begin{array}{l}\text { Study } \\
\text { CRC patients }\end{array}$} & $\begin{array}{c}\text { Evaluated } \\
\text { patients }\end{array}$ & Total PIPAC-OX & \multicolumn{3}{c}{ Response assessment } \\
\cline { 5 - 8 } & 17 & 14 & None & Partial & Major & Complete \\
\hline
\end{tabular}

CRC, colorectal cancer; PIPAC-OX, pressurized intraperitoneal aerosol chemotherapy with oxaliplatin.

Table 6 Cytological response

\begin{tabular}{|c|c|c|c|c|c|c|}
\hline Studies & CRC patients & Evaluated patients & Total PIPAC-OX & \multicolumn{3}{|c|}{ Response assessment } \\
\hline Ellebæk [2020] (17) & 24 & 15 & 75 & 2 & 8 & 5 \\
\hline Graversen [2020] (19) & 3 & 3 & 9 & 0 & 2 & 1 \\
\hline
\end{tabular}

$\mathrm{CRC}$, colorectal cancer; PIPAC-OX, pressurized intraperitoneal aerosol chemotherapy with oxaliplatin. 
Table 7 Macroscopic response

\begin{tabular}{lccccc}
\hline Studies & CRC patients & $\begin{array}{c}\text { Evaluated } \\
\text { patients }\end{array}$ & Total PIPAC-OX & \multicolumn{2}{c}{ Response assessment } \\
\cline { 3 - 6 } & 24 & 7 & 75 & Progressive & Stable \\
\hline Ellebæk [2020] (17) & 15 & 7 & 45 & $\mathrm{~ns}$ & $\mathrm{~ns}$ \\
Willaert [2019] (18) & 15 & 3 & 0 & 4 \\
\hline
\end{tabular}

$\mathrm{CRC}$, colorectal cancer; PIPAC-OX, pressurized intraperitoneal aerosol chemotherapy with oxaliplatin; ns, not shown.

Table 8 Biochemical response

\begin{tabular}{|c|c|c|c|c|c|c|}
\hline Study & CRC patients & $\begin{array}{l}\text { Evaluated } \\
\text { patients }\end{array}$ & Total PIPAC-OX & \multicolumn{3}{|c|}{ Response assessment } \\
\hline Willaert [2019] (18) & 15 & 14 & 45 & 2 & 2 & 6 \\
\hline
\end{tabular}

treated with PIPAC-OX monotherapy or PIPAC-OX with concomitant systemic therapy.

The other study reported that 2 of 3 patients were treated with PIPAC-OX with concomitant systemic therapy. However, the patient treated with PIPAC-OX monotherapy converted from positive to negative cytology, whereas the cytology of two patients treated with PIPAC-OX with concomitant systemic therapy remained stable in one patient and converted from negative to positive cytology in the other patient.

\section{Macroscopic response}

Macroscopic response was reported in five studies, but only two studies reported separate results for patients with CPM treated with PIPAC-OX (Table 7) $(17,18,21,27,36)$. The first study reported a decrease of ascites volume in 3 out of 7 (43\%) patients, but did not report on the other four patients (17). However, macroscopic response was not separately reported for patients receiving PIPAC-OX monotherapy and patients receiving PIPAC-OX with concomitant systemic therapy. The second study evaluated ascites volume in seven patients who had all been treated with PIPAC-OX with concomitant systemic therapy, and found a decrease in ascites volume in $4(57 \%)$ patients but an increase in $3(43 \%)$ patients (18).

\section{Biochemical response}

The biochemical response during treatment with PIPAC-
OX was reported in two studies $(18,20)$, but was only separately reported for patients with CPM in one study (Table 8) (18). Fourteen of 15 patients with CPM received at least two PIPAC-OX and underwent response evaluation. Tumor markers were not determined in four patients. In the other ten patients, tumor markers increased in $2(20 \%)$ patients, decreased in $6(60 \%)$ patients, and remained stable in $2(20 \%)$ patients. Out of two evaluable patients that did not receive PIPAC-OX with concomitant systemic therapy, tumor markers were not available in one patient and increased in the other patient. However, it was not mentioned how biochemical response and progression were defined and which tumor marker was used.

\section{Quality of life}

Although five studies reported quality of life results, none provided separate results for patients with CPM treated with PIPAC-OX $(20,28,33,35,40)$.

\section{PFS}

PFS was reported in two studies $(20,29)$, but only one reported separate outcomes for patients with CPM treated with PIPAC-OX (Table 9, A) (20). They reported a median PFS of 3 months in 16 patients with CPM that all received two or more PIPAC-OX. It was not reported from which time-point PFS was calculated. However, PFS was not separately reported for patients receiving PIPACOX monotherapy and patients receiving PIPAC-OX with 
Table 9 Progression-free and overall survival

\begin{tabular}{|c|c|c|c|c|c|c|c|}
\hline Studies & CRC patients & $\begin{array}{l}\text { Evaluated } \\
\text { patients }\end{array}$ & $\begin{array}{c}\text { Total } \\
\text { PIPAC-OX }\end{array}$ & $\begin{array}{l}\text { Median } \\
\text { (months) }\end{array}$ & 1 year & $\begin{array}{l}\text { Calculated } \\
\text { from }\end{array}$ & $\begin{array}{l}\text { Follow-up } \\
\text { (months) }\end{array}$ \\
\hline \multicolumn{8}{|c|}{ A. Progression-free survival } \\
\hline De Simone [2020] (20) & 23 & $16(b)$ & 32 (b) & 3 & ns & ns & ns \\
\hline \multicolumn{8}{|l|}{ B. Overall survival } \\
\hline De Simone [2020] (20) & 23 & $16(b)$ & 32 (b) & 27 & ns & ns & ns \\
\hline Kurtz [2018] (21) & 17 & 17 & ns & Not reached & $60 \%(c)$ & First PIPAC & $10 \pm 4(d)$ \\
\hline Sgarbura [2019] (22) & 66 & 66 & ns & Not reached & $67 \%(c)$ & First PIPAC & $5[5-11](e)$ \\
\hline
\end{tabular}

(b) Reported in patients that underwent at least 2 PIPAC; (c) as estimated from Kaplan-Meier survival curve; (d) mean \pm standard deviation; (e) median [interquartile range]. CRC, colorectal cancer; PIPAC-OX, pressurized intraperitoneal aerosol chemotherapy with oxaliplatin; ns, not shown.

Table 10 Eligibility for secondary cytoreductive surgery

\begin{tabular}{lcccc}
\hline Studies & CRC patients & Evaluated patients & Total PIPAC-OX & CRS performed \\
\hline Demtröder [2016] (15) & 17 & 17 & 42 & 2 \\
Ellebæk [2020] (17) & 24 & 24 & 75 & 0 \\
Alyami [2019] (25) & 31 & 31 & $\mathrm{~ns}$ & 0 \\
Girshally [2016] (26) & $\mathrm{ns}$ & $\mathrm{ns}$ & $\mathrm{ns}$ & 6 \\
\hline
\end{tabular}

CRS, cytoreductive surgery; CRC, colorectal cancer; PIPAC-OX, pressurized intraperitoneal aerosol chemotherapy with oxaliplatin; ns, not shown.

concomitant systemic therapy.

\section{OS}

OS was reported in eight studies $(15,17,20-22,27,29,33)$, and separately for patients with CPM treated with PIPACOX in five studies (Table 9, B). All five studies reported a median OS, which ranged from 15 to 27 months. OS was calculated from the first PIPAC-OX in four studies but the baseline time-point was not specified in one study. A median OS was not reached in two studies due to short follow-up. Four studies also showed Kaplan-Meier figures, of which a 1-year survival could be estimated, ranging from $60 \%$ to $67 \%$. However, none of these five studies separately reported survival outcomes for patients receiving PIPACOX monotherapy and PIPAC-OX with concomitant systemic therapy.

\section{Eligibility for cytoreductive surgery}

Six studies provided results of a subgroup of patients that were able to undergo cytoreductive surgery after treatment with PIPAC $(15,17,25,26,31,36)$, but two did not provide separate results for patients with CPM treated with PIPACOX (Table 10).

Two studies reported that no secondary cytoreductive surgery could be performed after repetitive PIPAC-OX in 54 patients with CPM $(17,25)$. In one study, 3 of 24 patients were treated with PIPAC-OX with concomitant systemic therapy, but the number of patients treated with PIPAC-OX monotherapy and PIPAC-OX with concomitant systemic therapy was not reported in the second study.

The third study performed secondary cytoreductive surgery in 2/17 (12\%) patients with CPM after treatment with PIPAC-OX (15). However, it was not described whether these patients had received PIPAC-OX 
monotherapy or PIPAC-OX with concomitant systemic therapy.

The fourth study reported that six patients with CPM received secondary cytoreductive surgery after repetitive treatment with PIPAC-OX (26). However, the total amount of patients with CPM treated with PIPAC-OX was not reported, thus it was not possible to extract a proportion of patients undergoing secondary cytoreductive surgery.

\section{Environmental safety}

Two studies investigated occupational exposure to oxaliplatin during PIPAC-OX for CPM $(23,24)$. Several samples were taken during two PIPAC-OX procedures. All samples showed undetectable concentrations of oxaliplatin in the air, surface wipes, or in surgeon's blood.

\section{Discussion}

This is the first systematic review providing an overview of the available evidence for the use of PIPAC-OX in patients with unresectable CPM. We found 28 clinical studies that included at least one patient with CPM treated with PIPAC-OX. Of these, 26 studies included patients receiving PIPAC with various drugs for various tumors, and only two solely focused on patients with CPM treated with PIPACOX. Of all 28 included studies, 14 studies provided at least one separate outcome for patients with CPM treated with PIPAC-OX. Limitations of these 14 studies were small colorectal cancer sample sizes, heterogeneous treatment regimens (PIPAC-OX monotherapy versus PIPAC-OX with concomitant systemic therapy) and heterogeneous outcome reporting (not stratifying for treatment regimen). Moreover, the majority of these studies reporting on survival did not stratify their results for patients receiving PIPAC-OX as first-line versus later-line treatment, and some studies reporting tumor response did not provide definitions of response and progression. Despite these limitations, the present systematic review shows that PIPAC-OX appears safe in patients with CPM and that no environmental exposure of oxaliplatin was detected during PIPAC-OX.

Five other studies have performed a systematic review of clinical studies on PIPAC for the treatment of peritoneal metastases (10,41-44). However, none of these systematic reviews specifically focused on PIPAC-OX in patients with CPM alone and reported on the interpretability of the results of included studies. Although these systematic reviews suggest that PIPAC is generally well tolerated, the present systematic review showed that the quality of life of patients with CPM treated with PIPAC-OX has never been reported. It is also generally assumed that PIPACOX is associated with low rates of systemic toxicity, whereas the present systematic review showed that the reporting of adverse events was not stratified for PIPACOX monotherapy and for PIPAC-OX with concomitant systemic therapy, which impedes the interpretation of these results. The lack of stratification for PIPAC-OX monotherapy and PIPAC-OX with concomitant systemic therapy also impedes the interpretation of other outcomes, such as tumor response, secondary curative intent surgery, and survival. Moreover, survival results were not stratified for line of palliative treatment, impeding comparison with survival results of trials on systemic therapy. Altogether, based on the available evidence consisting of noncomparative studies only, the (additional) benefit of PIPACOX for patients with CPM remains uncertain. Nevertheless, PIPAC-OX for colorectal cancer is currently increasingly practiced in multiple centers worldwide.

Thus, results from prospective cohorts or randomized controlled trials that provide outcomes stratified for primary tumor location, administration of PIPAC-OX monotherapy or PIPAC-OX with concomitant systemic therapy, and previous palliative systemic treatment are urgently required to gain more insight into these outcomes and to determine the exact role of PIPAC-OX in patients with CPM.

To the knowledge of the authors, there are currently two ongoing phase I dose escalation studies for PIPACOX $(45,46)$ and there are six ongoing phase II-III clinical studies investigating PIPAC-OX in patients with CPM (4749) (Netherlands Trial Register, NL8303; ClinicalTrials. gov, NCT04329494; NCT03868228). Three studies solely include patients with CPM and treat patients with PIPACOX monotherapy (47), PIPAC-OX with concomitant firstline systemic therapy (NL8303), or either PIPAC-OX monotherapy or PIPAC-OX with concomitant systemic therapy in any line of palliative treatment (NCT03868228). The other three studies include patients receiving PIPAC with various drugs for various origins, including PIPAC-OX for CPM. One of these studies is a randomized controlled trial, randomizing patients between PIPAC monotherapy and palliative systemic therapy (48). The other two studies treat patients with PIPAC monotherapy or PIPAC with concomitant systemic therapy (49) (NCT04329494). The investigators of the latter three studies are encouraged to report separate results for patients with CPM treated with PIPAC-OX to provide insights into the value of PIPAC-OX 
in this particular group.

\section{Conclusions}

Despite the increasing practice of PIPAC worldwide and the growing amount of available manuscripts on PIPAC, only very few studies focus on PIPAC-OX in patients with CPM or provide separate results for this subgroup. Therefore, the currently available evidence for the use of PIPAC-OX in patients with CPM is limited and difficult to interpret, mainly since the majority of studies did not stratify their results for PIPAC-OX monotherapy versus PIPAC-OX with concomitant systemic therapy. Investigators of future studies including patients receiving PIPAC-OX for CPM are encouraged to report separate outcomes for this particular group, to stratify their results for PIPAC-OX monotherapy versus PIPAC-OX with concomitant systemic therapy, and to stratify survival outcomes for line of palliative treatment. These results may help designing future randomized trials which are required to determine the exact role of PIPAC$\mathrm{OX}$ in patients with CPM.

\section{Acknowledgments}

Funding: None.

\section{Footnote}

Provenance and Peer Review: This article was commissioned by the Guest Editors (Paul H. Sugarbaker and Kurt Van der Speeten) for the focused issue "Intraperitoneal Chemotherapy for Peritoneal Metastases: HIPEC, EPIC, NIPEC, PIPAC and More" published in fournal of Gastrointestinal Oncology. This article has undergone external peer review.

Reporting Checklist: The authors have completed the PRISMA checklist. Available at http://dx.doi.org/10.21037/ jgo-20-257

Conflicts of Interest: All authors have completed the ICMJE uniform disclosure form (available at http://dx.doi. org/10.21037/jgo-20-257). The focused issue was sponsored by the Peritoneal Surface Oncology Group International (PSOGI). IHJH reports grants from ROCHE, grants from RanD Biotech, outside the submitted work. The authors have no other conflicts of interest to declare.
Ethical Statement: The authors are accountable for all aspects of the work in ensuring that questions related to the accuracy or integrity of any part of the work are appropriately investigated and resolved.

Open Access Statement: This is an Open Access article distributed in accordance with the Creative Commons Attribution-NonCommercial-NoDerivs 4.0 International License (CC BY-NC-ND 4.0), which permits the noncommercial replication and distribution of the article with the strict proviso that no changes or edits are made and the original work is properly cited (including links to both the formal publication through the relevant DOI and the license). See: https://creativecommons.org/licenses/by-nc-nd/4.0/.

\section{References}

1. Klaver YL, Simkens LH, Lemmens VE, et al. Outcomes of colorectal cancer patients with peritoneal carcinomatosis treated with chemotherapy with and without targeted therapy. Eur J Surg Oncol 2012;38:617-23.

2. Razenberg LG, Lemmens VE, Verwaal VJ, et al. Challenging the dogma of colorectal peritoneal metastases as an untreatable condition: Results of a population-based study. Eur J Cancer 2016;65:113-20.

3. Franko J, Shi Q, Meyers JP, et al. Prognosis of patients with peritoneal metastatic colorectal cancer given systemic therapy: an analysis of individual patient data from prospective randomised trials from the Analysis and Research in Cancers of the Digestive System (ARCAD) database. Lancet Oncol 2016;17:1709-19.

4. Jacquet P, Sugarbaker PH. Peritoneal-plasma barrier. Cancer Treat Res 1996;82:53-63.

5. Sugarbaker PH, Stuart OA, Vidal-Jove J, et al. Pharmacokinetics of the peritoneal-plasma barrier after systemic mitomycin C administration. Cancer Treat Res 1996;82:41-52.

6. Solaß W, Hetzel A, Nadiradze G, et al. Description of a novel approach for intraperitoneal drug delivery and the related device. Surg Endosc 2012;26:1849-55.

7. Solass W, Herbette A, Schwarz T, et al. Therapeutic approach of human peritoneal carcinomatosis with Dbait in combination with capnoperitoneum: proof of concept. Surg Endosc 2012;26:847-52.

8. Solass W, Kerb R, Murdter T, et al. Intraperitoneal chemotherapy of peritoneal carcinomatosis using pressurized aerosol as an alternative to liquid solution: first 
evidence for efficacy. Ann Surg Oncol 2014;21:553-9.

9. Blanco A, Giger-Pabst U, Solass W, et al. Renal and hepatic toxicities after pressurized intraperitoneal aerosol chemotherapy (PIPAC). Ann Surg Oncol 2013;20:2311-6.

10. Alyami M, Hubner M, Grass F, et al. Pressurised intraperitoneal aerosol chemotherapy: rationale, evidence, and potential indications. Lancet Oncol 2019;20:e368-77.

11. Nowacki M, Alyami M, Villeneuve L, et al. Multicenter comprehensive methodological and technical analysis of 832 pressurized intraperitoneal aerosol chemotherapy (PIPAC) interventions performed in 349 patients for peritoneal carcinomatosis treatment: An international survey study. Eur J Surg Oncol 2018;44:991-6.

12. Moher D, Liberati A, Tetzlaff J, et al. Preferred reporting items for systematic reviews and meta-analyses: the PRISMA statement. J Clin Epidemiol 2009;62:1006-12.

13. Siebert M, Alyami M, Mercier F, et al. Severe hypersensitivity reactions to platinum compounds postpressurized intraperitoneal aerosol chemotherapy (PIPAC): first literature report. Cancer Chemother Pharmacol 2019;83:425-30.

14. Katdare N, Prabhu R, Mishra S, et al. Pressurized Intraperitoneal Aerosol Chemotherapy (PIPAC): Initial Experience from Indian Centers and a Review of Literature. Indian J Surg Oncol 2019;10:24-30.

15. Demtröder C, Solass W, Zieren J, et al. Pressurized intraperitoneal aerosol chemotherapy with oxaliplatin in colorectal peritoneal metastasis. Colorectal Dis 2016;18:364-71.

16. Somashekhar SP, Ashwin KR, Kumar CR, et al. Pressurized intraperitoneal aerosol chemotherapy procedure for nonresectable peritoneal carcinomatosis: First Indian study. South Asian J Cancer 2019;8:27-30.

17. Ellebæk SB, Graversen M, Detlefsen S, et al. Pressurized IntraPeritoneal Aerosol Chemotherapy (PIPAC)-directed treatment of peritoneal metastasis in end-stage colo-rectal cancer patients. Pleura Peritoneum 2020;5:20200109.

18. Willaert W, Van de Sande L, Van Daele E, et al. Safety and preliminary efficacy of electrostatic precipitation during pressurized intraperitoneal aerosol chemotherapy (PIPAC) for unresectable carcinomatosis. Eur J Surg Oncol 2019;45:2302-9.

19. Graversen M, Detlefsen S, Ellebaek SB, et al. Pressurized IntraPeritoneal Aerosol Chemotherapy with one minute of electrostatic precipitation (ePIPAC) is feasible, but the histological tumor response in peritoneal metastasis is insufficient. Eur J Surg Oncol 2020;46:155-9.

20. De Simone M, Vaira M, Argenziano M, et al. Pressurized Intraperitoneal Aerosol Chemotherapy (PIPAC) with Oxaliplatin, Cisplatin, and Doxorubicin in Patients with Peritoneal Carcinomatosis: An Open-Label, Single-Arm, Phase II Clinical Trial. Biomedicines 2020;8:102.

21. Kurtz F, Struller F, Horvath P, et al. Feasibility, Safety, and Efficacy of Pressurized Intraperitoneal Aerosol Chemotherapy (PIPAC) for Peritoneal Metastasis: A Registry Study. Gastroenterol Res Pract 2018;2018:2743985.

22. Sgarbura O, Hubner M, Alyami M, et al. Oxaliplatin use in pressurized intraperitoneal aerosol chemotherapy (PIPAC) is safe and effective: A multicenter study. Eur J Surg Oncol 2019;45:2386-91.

23. Graversen M, Pedersen PB, Mortensen MB.

Environmental safety during the administration of Pressurized IntraPeritoneal Aerosol Chemotherapy (PIPAC). Pleura Peritoneum 2016;1:203-8.

24. Willaert W, Sessink P, Ceelen W. Occupational safety of pressurized intraperitoneal aerosol chemotherapy (PIPAC). Pleura Peritoneum 2017;2:121-8.

25. Alyami M, Mercier F, Siebert M, et al. Unresectable peritoneal metastasis treated by pressurized intraperitoneal aerosol chemotherapy (PIPAC) leading to cytoreductive surgery and hyperthermic intraperitoneal chemotherapy. Eur J Surg Oncol 2021;47:128-33.

26. Girshally R, Demtroder C, Albayrak N, et al. Pressurized intraperitoneal aerosol chemotherapy (PIPAC) as a neoadjuvant therapy before cytoreductive surgery and hyperthermic intraperitoneal chemotherapy. World J Surg Oncol 2016;14:253.

27. Kuchen N, Cereser T, Hailemariam S, et al. Safety and efficacy of pressurized intraperitoneal/intrathoracic aerosol chemotherapy (PIPAC/PITAC) in patients with peritoneal and/or pleural carcinomatosis: a preliminary experience. J Med Therap 2018;2:1-6.

28. Graversen M, Detlefsen S, Bjerregaard JK, et al.

Prospective, single-center implementation and response evaluation of pressurized intraperitoneal aerosol chemotherapy (PIPAC) for peritoneal metastasis. Ther Adv Med Oncol 2018;10:1758835918777036.

29. Benzerdjeb N, Durieux E, Tantot J, et al. Prognostic Impact of Combined Progression Index Based on Peritoneal Grading Regression Score and Peritoneal 
Cytology in Peritoneal Metastasis. Histopathology 2020;77:548-59.

30. Graversen M, Fristrup C, Kristensen TK, et al. Detection of free intraperitoneal tumour cells in peritoneal lavage fluid from patients with peritoneal metastasis before and after treatment with pressurised intraperitoneal aerosol chemotherapy (PIPAC). J Clin Pathol 2019;72:368-72.

31. Ceribelli C, Debs T, Chevallier A, et al. Initial experience of pressurized intraperitoneal aerosol chemotherapy (PIPAC) in a French hyperthermic intraperitoneal chemotherapy (HIPEC) expert center. Surg Endosc 2020;34:2803-6.

32. Teixeira Farinha H, Grass F, Labgaa I, et al. Inflammatory Response and Toxicity After Pressurized IntraPeritoneal Aerosol Chemotherapy. J Cancer 2018;9:13-20.

33. Odendahl K, Solass W, Demtroder C, et al. Quality of life of patients with end-stage peritoneal metastasis treated with Pressurized IntraPeritoneal Aerosol Chemotherapy (PIPAC). Eur J Surg Oncol 2015;41:1379-85.

34. Vaira M, Robella M, Borsano A, et al. Single-port access for Pressurized IntraPeritoneal Aerosol Chemotherapy (PIPAC): technique, feasibility and safety. Pleura Peritoneum 2016;1:217-22.

35. Robella M, Vaira M, De Simone M. Safety and feasibility of pressurized intraperitoneal aerosol chemotherapy (PIPAC) associated with systemic chemotherapy: an innovative approach to treat peritoneal carcinomatosis. World J Surg Oncol 2016;14:128.

36. Alyami M, Gagniere J, Sgarbura O, et al. Multicentric initial experience with the use of the pressurized intraperitoneal aerosol chemotherapy (PIPAC) in the management of unresectable peritoneal carcinomatosis. Eur J Surg Oncol 2017;43:2178-83.

37. Hübner M, Teixeira Farinha H, Grass F, et al. Feasibility and Safety of Pressurized Intraperitoneal Aerosol Chemotherapy for Peritoneal Carcinomatosis: A Retrospective Cohort Study. Gastroenterol Res Pract 2017;2017:6852749.

38. Graversen M, Lundell L, Fristrup C, et al. Pressurized IntraPeritoneal Aerosol Chemotherapy (PIPAC) as an outpatient procedure. Pleura Peritoneum 2018;3:20180128.

39. Siebert M, Alyami M, Mercier F, et al. Pressurized intraperitoneal aerosol chemotherapy (PIPAC) in association with systemic chemotherapy and bevacizumab, evaluation of safety and feasibility. A single center comparative study. Eur J Surg Oncol 2021;47:139-42.

40. Teixeira Farinha H, Grass F, Kefleyesus A, et al. Impact of Pressurized Intraperitoneal Aerosol Chemotherapy on Quality of Life and Symptoms in Patients with Peritoneal Carcinomatosis: A Retrospective Cohort Study. Gastroenterol Res Pract 2017;2017:4596176.

41. Grass F, Vuagniaux A, Teixeira-Farinha H, et al. Systematic review of pressurized intraperitoneal aerosol chemotherapy for the treatment of advanced peritoneal carcinomatosis. Br J Surg 2017;104:669-78.

42. Tempfer C, Giger-Pabst U, Hilal Z, et al. Pressurized intraperitoneal aerosol chemotherapy (PIPAC) for peritoneal carcinomatosis: systematic review of clinical and experimental evidence with special emphasis on ovarian cancer. Arch Gynecol Obstet 2018;298:243-57.

43. Winkler CS, Sandhu J, Pettke E, et al. Pressurized Intraperitoneal Aerosol Chemotherapy, a Palliative Treatment Approach for Patients With Peritoneal Carcinomatosis: Description of Method and Systematic Review of Literature. Dis Colon Rectum 2020;63:242-55.

44. Tate SJ, Torkington J. Pressurized intraperitoneal aerosol chemotherapy: a review of the introduction of a new surgical technology using the IDEAL framework. BJS Open 2020;4:206-15.

45. Kim G, Tan HL, Chen E, et al. Study protocol: phase 1 dose escalating study of Pressurized IntraPeritoneal Aerosol Chemotherapy (PIPAC) with oxaliplatin in peritoneal metastasis. Pleura Peritoneum 2018;3:20180118.

46. Dumont F, Senellart H, Pein F, et al. Phase I/II study of oxaliplatin dose escalation via a laparoscopic approach using pressurized aerosol intraperitoneal chemotherapy (PIPOX trial) for nonresectable peritoneal metastases of digestive cancers (stomach, small bowel and colorectal): Rationale and design. Pleura Peritoneum 2018;3:20180120.

47. Rovers KP, Lurvink RJ, Wassenaar EC, et al. Repetitive electrostatic pressurised intraperitoneal aerosol chemotherapy (ePIPAC) with oxaliplatin as a palliative monotherapy for isolated unresectable colorectal peritoneal metastases: protocol of a Dutch, multicentre, open-label, single-arm, phase II study (CRC-PIPAC). BMJ Open 2019;9:e030408.

48. Somashekhar SP, Ashwin KR, Rauthan CA, et al. Randomized control trial comparing quality of life of patients with end-stage peritoneal metastasis treated with pressurized intraperitoneal aerosol chemotherapy (PIPAC) 
and intravenous chemotherapy. Pleura Peritoneum 2018;3:20180110.

49. Graversen M, Detlefsen S, Asmussen J, et al. Treatment of peritoneal carcinomatosis with Pressurized IntraPeritoneal Aerosol Chemotherapy - PIPAC-OPC2. Pleura

Peritoneum 2018;3:20180108.

Cite this article as: Lurvink RJ, Rovers KP, Nienhuijs SW, Creemers GJ, Burger JWA, de Hingh IHJ. Pressurized intraperitoneal aerosol chemotherapy with oxaliplatin (PIPAC$\mathrm{OX}$ ) in patients with colorectal peritoneal metastasesa systematic review. J Gastrointest Oncol 2021;12(Suppl 1): S242-S258. doi: 10.21037/jgo-20-257 


\section{Appendix A-Search}

\section{Pubmed search}

((PIPAC) or (Pressurized Intraperitoneal Aerosol Chemotherapy) OR (Pressurised Intraperitoneal Aerosol Chemotherapy))

\section{Appendix B-Details of excluded clinical studies}

\section{Study on PIPAC-cisplatin-doxorubicin for non-CPM}

1. Blanco A, Giger-Pabst U, Solass W, Zieren J, Reymond MA. Renal and hepatic toxicities after pressurized intraperitoneal aerosol chemotherapy (PIPAC). Ann Surg Oncol 2013;20:2311-6.

2. Solass W, Kerb R, Mürdter T, et al. Intraperitoneal chemotherapy of peritoneal carcinomatosis using pressurized aerosol as an alternative to liquid solution: first evidence for efficacy. Ann Surg Oncol 2014;21:553-9.

3. Tempfer CB, Celik I, Solass W, et al. Activity of Pressurized Intraperitoneal Aerosol Chemotherapy (PIPAC) with cisplatin and doxorubicin in women with recurrent, platinum-resistant ovarian cancer: preliminary clinical experience. Gynecol Oncol 2014;132:307-11.

4. Tempfer CB, Winnekendonk G, Solass W, et al. Pressurized intraperitoneal aerosol chemotherapy in women with recurrent ovarian cancer: A phase 2 study. Gynecol Oncol 2015;137:223-8.

5. Giger-Pabst U, Solass W, Buerkle B, et al. Low-dose pressurized intraperitoneal aerosol chemotherapy (PIPAC) as an alternative therapy for ovarian cancer in an octogenarian patient. Anticancer Res 2015;35:2309-14.

6. Tempfer CB, Solass W, Buerkle B, et al. Pressurized intraperitoneal aerosol chemotherapy (PIPAC) with cisplatin and doxorubicin in a woman with pseudomyxoma peritonei: A case report. Gynecol Oncol Rep 2014;10:32-5.

7. Nadiradze G, Giger-Pabst U, Zieren J, et al. Pressurized Intraperitoneal Aerosol Chemotherapy (PIPAC) with LowDose Cisplatin and Doxorubicin in Gastric Peritoneal Metastasis. J Gastrointest Surg 2016;20:367-73.

8. Tempfer CB, Rezniczek GA, Ende P, et al. Pressurized Intraperitoneal Aerosol Chemotherapy with Cisplatin and Doxorubicin in Women with Peritoneal Carcinomatosis: A Cohort Study. Anticancer Res 2015;35:6723-9.

9. Reymond M, Demtroeder C, Solass W, et al. Electrostatic precipitation Pressurized IntraPeritoneal Aerosol
Chemotherapy (ePIPAC): first in-human application. Pleura Peritoneum 2016;1:109-16.

10. Rezniczek GA, Jüngst F, Jütte $H$, et al. Dynamic changes of tumor gene expression during repeated pressurized intraperitoneal aerosol chemotherapy (PIPAC) in women with peritoneal cancer. BMC Cancer 2016;16:654.

11. Khomyakov V, Ryabov A, Ivanov A, et al. Bidirectional chemotherapy in gastric cancer with peritoneal metastasis combining intravenous XELOX with intraperitoneal chemotherapy with low-dose cisplatin and Doxorubicin administered as a pressurized aerosol: an open-label, Phase-2 study (PIPAC-GA2). Pleura Peritoneum 2016;1:159-66.

12. Tempfer CB, Hartmann F, Hilal Z, et al. Intraperitoneal cisplatin and doxorubicin as maintenance chemotherapy for unresectable ovarian cancer: a case report. BMC Cancer 2017;17:26.

13. Graversen M, Detlefsen S, Bjerregaard JK, et al. Peritoneal metastasis from pancreatic cancer treated with pressurized intraperitoneal aerosol chemotherapy (PIPAC). Clin Exp Metastasis 2017;34:309-14.

14. Sleeman JP. PIPAC puts pressure on peritoneal metastases from pancreatic cancer. Clin Exp Metastasis 2017;34:291-3.

15. Hilal Z, Rezniczek GA, Klenke R, et al. Nutritional status, cachexia, and anorexia in women with peritoneal metastasis and intraperitoneal chemotherapy: a longitudinal analysis. J Gynecol Oncol 2017;28:e80.

16. Khosrawipour T, Khosrawipour V, Giger-Pabst U. Pressurized Intra Peritoneal Aerosol Chemotherapy in patients suffering from peritoneal carcinomatosis of pancreatic adenocarcinoma. PLoS One 2017;12:e0186709.

17. Falkenstein TA, Götze TO, Ouaissi M, et al. First Clinical Data of Pressurized Intraperitoneal Aerosol Chemotherapy (PIPAC) as Salvage Therapy for Peritoneal Metastatic Biliary Tract Cancer. Anticancer Res 2018;38:373-8.

18. Giger-Pabst U, Demtröder C, Falkenstein TA, et al. Pressurized IntraPeritoneal Aerosol Chemotherapy (PIPAC) for the treatment of malignant mesothelioma. BMC Cancer 2018;18:442.

19. Somashekhar SP, Rajagopal AK, Zaveri SS, et al. First Indian study on pressurized intraperitoneal aerosol chemotherapy (PIPAC) procedure for advanced peritoneal carcinomatosis secondary to epithelial ovarian cancer. Indian J Gynecol Oncolog. 2018;16:25.

20. Tempfer CB, Giger-Pabst U, Seebacher V, et al. A 
phase I, single-arm, open-label, dose escalation study of intraperitoneal cisplatin and doxorubicin in patients with recurrent ovarian cancer and peritoneal carcinomatosis. Gynecol Oncol 2018;150:23-30.

21. Zakharenko AA, Belyaev MA, Trushiti AA, et al. First results of laparoscopic pressurized intreaperitoneal aerosol chemotherapy (PIPAC) in treatment for peritoneal carcinomatosis in patients with gastric cancer. Voprosy Onkologii 2018;64:222-7.

22. Tempfer CB, Hilal Z, Dogan A, et al. Concentrations of cisplatin and doxorubicin in ascites and peritoneal tumor nodules before and after pressurized intraperitoneal aerosol chemotherapy (PIPAC) in patients with peritoneal metastasis. Eur J Surg Oncol 2018;44:1112-7.

23. Nowacki M, Grzanka D, Zegarski W. Pressurized intraperitoneal aerosol chemotheprapy after misdiagnosed gastric cancer: Case report and review of the literature. World J Gastroenterol 2018;24:2130-6.

24. Horvath P, Beckert S, Struller F, et al. Pressurized intraperitoneal aerosol chemotherapy (PIPAC) for peritoneal metastases of pancreas and biliary tract cancer. Clin Exp Metastasis 2018;35:635-40.

25. Larbre V, Alyami M, Mercier F, et al. No Renal Toxicity After Repeated Treatment with Pressurized Intraperitoneal Aerosol Chemotherapy (PIPAC) in Patients with Unresectable Peritoneal Metastasis. Anticancer Res 2018;38:6869-75.

26. Gockel I, Jansen-Winkeln B, Haase L, et al. Pressurized Intraperitoneal Aerosol Chemotherapy (PIPAC) in Gastric Cancer Patients with Peritoneal Metastasis (PM): Results of a Single-Center Experience and Register Study. J Gastric Cancer. 2018;18:379-91.

27. Struller F, Horvath P, Solass W, et al. Pressurized intraperitoneal aerosol chemotherapy with low-dose cisplatin and doxorubicin (PIPAC C/D) in patients with gastric cancer and peritoneal metastasis: a phase II study. Ther Adv Med Oncol 2019;11:1758835919846402.

28. Nowacki M, Nowacka K, Głowacka I, et al. Overall clinical and trichoscopic analysis performed in patients who underwent pressurized intraperitoneal aerosol chemotherapy (PIPAC) treatment for peritoneal carcinomatosis - initial trial preliminary report. Postepy Dermatol Alergol 2019;36:461-7.

29. Ellebæk SB, Graversen M, Detlefsen S, et al. Pressurized intraperitoneal aerosol chemotherapy (PIPAC) of peritoneal metastasis from gastric cancer: a descriptive cohort study. Clin Exp Metastasis 2020;37:325-32.
30. Nielsen M, Graversen M, Ellebæk SB, et al. Nextgeneration sequencing and histological response assessment in peritoneal metastasis from pancreatic cancer treated with PIPAC. J Clin Pathol 2020. [Epub ahead of print]. doi:10.1136/jclinpath-2020-206607.

31. Alyami M, Bonnot PE, Mercier F, et al. Pressurized intraperitoneal aerosol chemotherapy (PIPAC) for unresectable peritoneal metastasis from gastric cancer. Eur J Surg Oncol 2020. [Epub ahead of print]. doi:10.1016/ j.ejso.2020.05.021.

32. Ndaw S, Hanser O, Kenepekian V, et al. Occupational exposure to platinum drugs during intraperitoneal chemotherapy. Biomonitoring and surface contamination. Toxicol Lett 2018;298:171-6.

33. Ametsbichler P, Böhlandt A, Nowak D, et al. Occupational exposure to cisplatin/oxaliplatin during Pressurized Intraperitoneal Aerosol Chemotherapy (PIPAC)? Eur J Surg Oncol 2018;44:1793-9.

34. Delhorme JB, Klipfel A, D'Antonio F, et al. Occupational safety of pressurized intraperitoneal aerosol chemotherapy (PIPAC) in an operating room without laminar airflow. J Visc Surg 2019;156:485-8.

35. Solass W, Giger-Pabst U, Zieren J, et al. Pressurized intraperitoneal aerosol chemotherapy (PIPAC): occupational health and safety aspects. Ann Surg Oncol 2013;20:3504-11.

36. Oyais A, Solass W, Zieren J, et al. Occupational Health Aspects of Pressurised Intraperitoneal Aerosol Chemotherapy (PIPAC): Confirmation of Harmlessness. Zentralbl Chir 2016;141:421-4.

\section{Study on PIPAC-cisplatin-doxorubicin for various indications, including CPM $(n=2)$}

1. Jansen-Winkeln B, Thieme R, Haase L, et al. Perioperative safety of intraperitoneal aerosol chemotherapy : Analysis of our first 111 pressurized intraperitoneal aerosol chemotherapy (PIPAC) procedures. Chirurg 2019;90:137-45.

2. Gockel I, Jansen-Winkeln B, Haase L, et al. Pressurized IntraPeritoneal Aerosol Chemotherapy (PIPAC) in patients with peritoneal metastasized colorectal, appendiceal and small bowel cancer. Tumori 2020;106:70-8.

\section{Study on PIPAC-oxaliplatin for non-CPM $(n=1)$}

1. Graversen M, Detlefsen S, Pfeiffer P, et al. Severe 
peritoneal sclerosis after repeated pressurized

intraperitoneal aerosol chemotherapy with oxaliplatin

(PIPAC OX): report of two cases and literature survey.

Clin Exp Metastasis 2018;35:103-8.

Study on procedural characteristics of PIPAC with various drugs for various indications $(n=1)$

1. Hübner M, Grass F, Teixeira-Farinha H, et a;. Pressurized intraperitoneal aerosol chemotherapy - practical aspects.

Eur J Surg Oncol 2017;43:1102-9.

\section{Study on PIPAC in solid organ transplant recipients $(n=1)$}

1. Horvath P, Yurttas C, Struller F, et al. Pressurized intraperitoneal aerosol chemotherapy (PIPAC) for peritoneal metastases in solid organ graft recipients: first experience. Ann Transplant 2019;24:30-5.
Study on pharmacokinetics of PIPAC-oxaliplatin for CPM $(n=1)$

1. Lurvink RJ, Tajzai R, Rovers KP, et al. Systemic Pharmacokinetics of Oxaliplatin After Intraperitoneal Administration by Electrostatic Pressurized Intraperitoneal Aerosol Chemotherapy (ePIPAC) in Patients with Unresectable Colorectal Peritoneal Metastases in the CRC-PIPAC Trial. Ann Surg Oncol 2020. [Epub ahead of print]. doi:10.1245/s10434-020-08743-9.

\section{Study on anesthesiological concerns and management during PIPAC (n=1)}

1. Solanki SL, Kumar PP, DeSouza A, Salkani AP.

Perioperative concerns and management of pressurised intraperitoneal aerosolised chemotherapy: report of two cases. Indian J Anaesth 2018;62:225-8. 